\title{
Chronic administration of Eucommia leaf stimulates metabolic function of rats across several organs
}

\author{
Takahiko Fujikawa ${ }^{1,2}$, Tetsuya Hirata $^{3}$, Atsunori Wada $^{3}$, Naomi Kawamura ${ }^{3}$, Yasuyo Yamaguchi ${ }^{3}$, \\ Katsuyuki Fujimura ${ }^{3}$, Taro Ueda ${ }^{3}$, Yutaka Yurugi ${ }^{4}$, Hideaki Soya ${ }^{5}$ and Sansei Nishibe ${ }^{6}$ \\ ${ }^{1}$ Laboratory of Molecular Prophylaxis and Pharmacology, Faculty of Pharmaceutical Sciences, \\ Suzuka University of Medical Science, 3500-3 Minamitamagaki-cho, Susuka, Mie 513-8670, Japan \\ ${ }^{2}$ Department of Biochemistry and Proteomics, Mie University Graduate School of Medicine, \\ 2-174 Edobashi, Tsu, Mie 514-8507, Japan \\ ${ }^{3} R \& D$ Center, Kobayashi Pharmaceutical Company, Limited, 1-30-3 Toyokawa, Ibaraki, Osaka 567-0057, Japan \\ ${ }^{4}$ Field Science Center of Kii Kuroshio Life Area, Mie University Graduate School of Bioresources, \\ 1577 Kurimamachiya, Tsu, Mie 514 -8507, Japan \\ ${ }^{5}$ Laboratory of Exercise Biochemistry, Graduate School of Comprehensive Human Sciences, University of Tsukuba, \\ 1-1-1 Tennoudai, Tsukuba, Ibaraki 305-8574, Japan \\ ${ }^{6}$ Department of Pharmacognosy, Faculty of Pharmaceutical Sciences, Health Sciences University of Hokkaido, 1757 Kanazawa, \\ Tobetsu-cho, Ishikari, Hokkaido 061-0293, Japan
}

(Received 17 January 2010 - Revised 7 June 2010 - Accepted 10 June 2010 - First published online 9 August 2010)

Eucommia bark (Eucommia ulmoides Oliver) has been used as an herbal medicine, and more recently, the plant's leaves have been widely used to prepare tea which may have anti-obesity properties. We used a metabolic syndrome-like rat model, produced by feeding a $35 \%$ high-fat diet (HFD), to examine potential anti-obesity and anti-metabolic syndrome effects and mechanisms of chronic administration of Eucommia leaf as an extract or green leaf powder. Eighty rats were studied for 3 months in ten groups. Both forms of Eucommia leaves minimised increases in body weight and visceral fat in a dose-dependent fashion. Increases in plasma levels of TAG and NEFA, and insulin resistance secondary to HFD were lessened by both forms of Eucommia leaf. Concomitantly, an increase in plasma adiponectin levels and suppression of plasma resistin and TNF- $\alpha$ levels were confirmed. Real-time PCR studies showed that both forms of Eucommia leaf enhanced metabolic function across several organs, including diminishing ATP production (white adipose tissue), accelerating $\beta$-oxidation (liver) and increasing the use of ketone bodies/ glucose (skeletal muscle), all of which may exert anti-obesity effects under HFD conditions. These findings suggest that chronic administration of either form of Eucommia leaves stimulates the metabolic function in rats across several organs. The anti-obesity and anti-metabolic syndrome activity in this rat model may be maintained through secretion and regulation of adipocytokines that depend on the accumulation of visceral fat to improve insulin resistance or hyperlipaemia.

Eucommia leaf: Chronic administration: Anti-obesity effects: Metabolic functions: Real-time PCR

Eucommia bark, Eucommia ulmoides Oliver (Eucommiaceae), is an herbal medicine traditionally used as an analeptic and analgesic, sedative, anti-hypertensive or diuretic medication ${ }^{(1)}$. The commercial product termed 'Tochu-cha' in Japanese is composed of the roasted leaves of Eucommia. Eucommia leaves have also been used in foods and contain the following active ingredients ${ }^{(2,3)}$ : geniposidic acid and asperuloside as iridoid glycosides, and chlorogenic acid as a coffeic acid derivative. From the green leaves of this plant, three new iridoids have been isolated, together with twelve known compounds ${ }^{(4,5)}$. Since the 1970 s, Eucommia leaf has been used in the Sichuan District of China as an anti-hypertensive drug or health food ${ }^{(6,7)}$, and clinical studies have been conducted to evaluate its efficacy ${ }^{(8-10)}$. Namba et al. carried out preliminary pharmacological studies of a water-based extract of Eucommia leaf and reported that a transient anti-hypertensive effect was exerted due to an agonistic effect on the parasympathetic nervous system ${ }^{(11,12)}$. Oral feeding of ad libitum Eucommia leaf extract (ELE) yielded dose-dependent antihypertensive effects in the spontaneously hypertensive rat $^{(13)}$. A follow-up study on the anti-hypertensive effect of ELE and its components in spontaneously hypertensive rat supported the findings of the initial study ${ }^{(14)}$.

ELE has also been reported to have potent antioxidant and anti-mutagenic effects ${ }^{(15)}$, and to prevent oxidative gastric injury $^{(16)}$. Recently, Fujikawa et al. ${ }^{(17)}$ found that ELE and

Abbreviations: BAT, brown adipose tissue; Cont., control; EGLP, Eucommia green leaf powder; ELE, Eucommia leaf extract; HFD, high-fat diet; ND, normal diet;

UCP, uncoupling protein (ATP synthase); WAT, white adipose tissue.

*Corresponding author: Dr T. Fujikawa, fax +81 59368 1271, email fujikawa@suzuka-u.ac.jp; t-fuji@doc.medic.mie-u.ac.jp 
its components may have prophylactic effects against restraint stress in water-induced gastric erosion. Ando et al. ${ }^{(18)}$ reported that the anti-obesity effects of the ELE are similar to those of the powdered leaf.

Obesity, which can lead to the metabolic syndrome, has recently gained attention as a factor that dramatically increases the risk of arteriosclerotic disease. According to Japanese diagnostic criteria, obesity and the metabolic syndrome are considered conditions requiring prevention and/or treatment due to the high risk of complications, including hyperglycaemia, hyperlipaemia and hypertension, based on accumulation of visceral fat. It has also been reported that accumulated visceral fat tissues secrete various types of adipocytokines and cause metabolic abnormalities such as insulin resistance ${ }^{(19)}$. Since in many cases the metabolic syndrome is triggered by a lack of exercise or a high-fat diet (HFD), basic treatment of it involves improvement of these lifestyle habits. Previous studies examined the effects on rats of high-dose administration of Eucommia leaf for $35 \mathrm{~d}^{(20)}$ and on hamsters of low-dose administration for 10 weeks ${ }^{(21)}$. Moreover, though the ELE is used for health maintenance in Japan, any anti-obesity or anti-metabolic-syndrome effects and mechanisms of chronic administration of Eucommia leaf in animals have yet to be fully elucidated.

The aim of the present study was to examine potential anti-obesity and anti-metabolic syndrome effects and mechanisms of chronic administration of Eucommia leaf as an extract or green leaf powder. Furthermore, a real-time PCR study examined potential metabolic effects across several organs including the perirenal white adipose tissue (WAT), liver, soleus muscle and brown adipose tissue (BAT) in rats when either form of Eucommia leaves was administered.

\section{Materials and methods}

\section{Animals and diets}

Male Sprague-Dawley rats (4 weeks old; 75-80 g) purchased from SLC, Incorporation (Shizuoka, Japan) were maintained at a temperature of $23-26^{\circ} \mathrm{C}$ and relative humidity of $50-65 \%$ for 2 weeks after arrival. Rats were divided into ten groups (normal diet (ND)-control (Cont.), ND-3\%-ELE, ND-9\%-ELE, ND-3\%-Eucommia green leaf powder (EGLP), ND-9\%-EGLP, HFDCont., HFD-3\%ELE, HFD$9 \%$ ELE, HFD-3 \%EGLP, and HFD-9\%EGLP; $n$ 8) based on their body weight. Under ND $(1506 \cdot 2 \mathrm{~kJ}(360 \mathrm{kcal}) / 100 \mathrm{~g})$ conditions, rats fed the commercial diet (Oriental Yeast Company, Limited, Tokyo, Japan) containing water $7.7 \%$, protein $23.6 \%$, lipid $5.3 \%$, ash $6.1 \%$, dietary fibre (measured by the Prosky method (Association of Analytical Chemists)) $2.9 \%$ and nitrogen-free extract (calculated as \%nitrogen-free extract $=100-($ water + protein + lipid + ash + dietary

fibre) $54.4 \%$. Under HFD $(2635.9 \mathrm{~kJ}(630 \mathrm{kcal}) / 100 \mathrm{~g})$ conditions, all the rats were fed the commercial diet with $29.7 \%$ lard (Oriental Yeast Company, Limited, Tokyo, Japan) containing water $7.7 \%$, protein $23.6 \%$, lipid $35.0 \%$, ash $6.1 \%$, dietary fibre $2.9 \%$ and nitrogen-free extract $24.7 \%$. Test foods were prepared by adding ELE and EGLP at 3 and $9 \%$, respectively, or by adding $9 \%$ casein as a control to determine the effects of a high-dose administration in rats, which were given from the outset of the experiment, ad libitum, for 3 months to examine potential anti-obesity and anti-metabolic syndrome effects of both forms of Eucommia leaves. These doses in rats fed a HFD were set referring to the findings of Metori et. $a l^{(20)}$ and corresponded to doses of $0.99-2 \cdot 35 \mathrm{~g} / \mathrm{d}$ (human subjects of body weight $60 \mathrm{~kg}$ and energy intake $8368 \mathrm{~kJ} / \mathrm{d}(2000 \mathrm{kcal} / \mathrm{d}))$. After chronic administration of Eucommia leaves, body weight was measured, and rats were killed by decapitation without stress. WAT (perirenal WAT and epididymal WAT), BAT and skeletal muscle (soleus muscle of hind leg) were immediately removed.

The Institutional Animal Care and Use Committees at Mie University Faculty of Medicine and the Suzuka University of Medical Science approved the animal facilities and study protocols. All procedures accorded the National Institutes of Health's guidelines regarding the principles of animal care (1996).

\section{Preparation of Eucommia leaf extract and Eucommia green leaf powder}

Eucommia leaf (E. ulmoides Oliver) collected in the Sichuan District of China was used. ELE was prepared as follows: fresh Eucommia leaves were treated with steam at 100 $110^{\circ} \mathrm{C}$, and then dried and roasted. Two tons of roasted Eucommia leaves were steeped in 10 tons of hot water at $90^{\circ} \mathrm{C}$ for $1 \mathrm{~h}$, and the extract was filtered and concentrated. The concentrate was left to stand for $1 \mathrm{~d}$. It was filtered and concentrated again, vacuum-dried and powdered (yield: $18 \%$ ). EGLP was prepared as follows: fresh Eucommia leaves were treated with steam at $100-110^{\circ} \mathrm{C}$ and dried, parched with far-IR radiation with a ceramic heater, and then pulverised with a jet mill.

We measured each of the major components (geniposidic acid, asperuloside and chlorogenic acid) in each sample using HPLC with an ODS system column ${ }^{(11)}$. ELE was comprised of geniposidic acid $69.5 \mathrm{mg} / \mathrm{g}$, asperuloside $13.7 \mathrm{mg} / \mathrm{g}$ and chlorogenic acid $34.4 \mathrm{mg} / \mathrm{g}$, while EGLP was comprised of geniposidic acid $31.8 \mathrm{mg} / \mathrm{g}$, asperuloside $27.8 \mathrm{mg} / \mathrm{g}$ and chlorogenic acid $17.8 \mathrm{mg} / \mathrm{g}$.

\section{Body weight, food intake and weight of white adipose tissue and skeletal muscle}

Body weight and food intake were measured once weekly throughout the experimental period. After total weekly consumption was measured, each rat's average daily food consumption was determined. After killing of rats, perirenal WAT, epididymal WAT and the soleus muscle of the hind leg were removed, and their wet weights were measured. Weights were calculated as ratios of wet weight to body weight.

\section{Blood components}

Blood was collected immediately after the animals were killed by decapitation. The blood was centrifuged $(3000 \mathrm{rpm}$ and $30 \mathrm{~min}$ ), and the separated plasma was stored at $-80^{\circ} \mathrm{C}$ until measurement. Plasma glucose and insulin were measured as biomarkers of carbohydrate metabolism, and total cholesterol, TAG and NEFA in plasma were measured as biomarkers of lipid metabolism. Plasma levels of adipocytokines (adiponectin, TNF- $\alpha$, resistin and leptin) were also measured. 
Insulin levels were measured by ELISA using an ultra highsensitive rat insulin assay kit (Morinaga Milk Industry Company, Limited, Tokyo, Japan), adiponectin was measured using a mouse/rat adiponectin ELISA kit (Otsuka Pharmaceutical Company, Limited, Tokyo, Japan), TNF- $\alpha$ was measured using a rat TNF- $\alpha$ assay kit (Immuno-Biological Laboratories Company, Limited, Gunma, Japan), resistin was measured using a Resistin ELISA kit (BioVender Laboratory Medicine, Brno, Czech Republic) and leptin was measured using a Leptin ELISA kit (BioVender Laboratory Medicine). Other parameters were measured using a Hitachi 7180 automated analyser.

\section{Real-time PCR}

Total RNA was extracted using the TriPure Isolation kit (Roche, Tokyo, Japan), and RNA concentrations were determined spectrophotometrically at $260 \mathrm{~nm}$. The quality of RNA was checked spectrophotometrically using the ratio of absorbances at $260 / 280 \mathrm{~nm}$, and the values of all the samples were found to be within the range of 1.8-2.0. After extraction, the RNA samples were treated with TURBO DNase free (Ambion, Austin, TX, USA) for $30 \mathrm{~min}$ at $37^{\circ} \mathrm{C}$ to remove any genomic DNA. The DNase-treated RNA $(0 \cdot 35 \mu \mathrm{g})$ was used to synthesise first-strand complementary DNA using the ReverTraAce qPCR RT kit (Toyobo, Tokyo, Japan). The complementary DNA products $(50 \mathrm{ng} / \mu \mathrm{l})$ were analysed by real-time PCR using Power SYBR Green PCR Master Mix (Applied Biosystems, Tokyo, Japan) in an ABI PRISM 7300 (Applied Biosystems). The amplification programme included an initial denaturation step at $95^{\circ} \mathrm{C}$ for $10 \mathrm{~min}$, forty cycles of denaturation at $95^{\circ} \mathrm{C}$ for $15 \mathrm{~s}$ and annealing/extension at $59-60^{\circ} \mathrm{C}$ for $1 \mathrm{~min}$. The level of glyceraldehyde-3-phosphate dehydrogenase mRNA was estimated for use as an internal control. Each gene was normalised to glyceraldehyde-3-phosphate dehydrogenase by subtracting the cycle threshold $(C \mathrm{t})$ value of glyceraldehyde-3-phosphate dehydrogenase from the $C \mathrm{t}$ value of the gene target ( $\Delta C \mathrm{t}$ (target)). Relative expression of the target gene was calculated using SDS v1.2 with relative quantification software (Applied Biosystems), with $\Delta C \mathrm{t}$ (target)s compared with $\Delta C \mathrm{t}$ values for reference; i.e. $\Delta \Delta C \mathrm{t}=\Delta C \mathrm{t}($ target $)-\Delta C \mathrm{t}$ (reference). The degree of difference (expressed in fold difference) between the target and the reference was calculated as $2^{-\Delta \Delta C t}$. Melting point analysis detected no non-specific amplification in the complementary DNA samples. The slopes of amplification curves did not differ between the groups on mRNA analysis, and no differences in amplification efficiency were observed. The sequences of the specific primers used in the Power SYBR Green Master Mix protocol are given in the supplementary Table. Each PCR primer was designed using DINASIS Pro v2.7 software (Hitachi software Engineering Company, Limited, Tokyo, Japan), and the oligonucleotides were purchased from Greiner Bio-One Company, Limited (Tokyo, Japan). The primers, Tm $\left({ }^{\circ} \mathrm{C}\right)$ and products (bp) of real-time PCR using the Power SYBR Green PCR Master Mix protocol are shown in the supplementary Table S1 (available online only at http://journals. cambridge.org).

\section{Statistical analysis}

All values are presented as the means with their standard errors for a given number of observation $(n)$. Differences between the groups were assessed with the unpaired Student's $t$ test or Tukey's honestly significant difference test using a statistical package from the SPSS13.0 program. Differences were considered significant at $P<0 \cdot 05$.

\section{Results}

Comparison of normal diet-control group and high-fat dietcontrol group

Comparison of the ND-Cont. group and the HFD-Cont. group revealed that rats fed a HFD for 3 months exhibited an increase in body weight $(P<0 \cdot 01)$ as well as a significant accumulation of visceral fat $(P<0 \cdot 001$, Table 1$)$. Concomitantly, an increase in energy intake (mean value: ND, $577 \mathrm{~kJ}(137.9 \mathrm{kcal}) / \mathrm{d}$; HFD, $666.9 \mathrm{~kJ}(159.4 \mathrm{kcal}) / \mathrm{d})$ was confirmed, although food intake decreased in rats fed a HFD $(P<0 \cdot 05$, Table 1$)$. Among blood indices in rats fed a HFD, a marked increase in plasma insulin level $(P<0 \cdot 001)$, a significant decrease in plasma total cholesterol level $(P<0.05)$ and a tendency towards increased plasma levels of TAG and NEFA were found (both $P<0 \cdot 1$, Table 1 ). Among adipocytokines, significant increases in plasma resistin and leptin levels $(P<0.05$ and 0.01 , respectively) and a decrease in plasma adiponectin level $(P<0 \cdot 1)$ were observed in rats fed a HFD for 3 months, while their plasma TNF- $\alpha$ levels $(P<0 \cdot 01)$ decreased significantly (Table 1).

Body weight, food intake and white adipose tissue weight with chronic administration of Eucommia leaf extract and Eucommia green leaf powder

Rats fed a ND supplemented with 3 or $9 \%$ of ELE and EGLP (ND-3\%ELE, ND-9\%ELE, ND-3\%EGLP and ND$9 \%$ EGLP) exhibited a tendency towards decreased body weight in 3 months compared with the ND-Cont. group (each $P<0 \cdot 1$, Table 2). Rats fed a HFD supplemented with 3 or $9 \%$ of either form of Eucommia leaves (HFD-3\%ELE,

Table 1. Comparison between normal diet control (ND-Cont.) and high-fat diet control (HFD-Cont.) after 90-d administration

(Mean values with their standard errors)

\begin{tabular}{|c|c|c|c|c|}
\hline \multirow[b]{2}{*}{ Item } & \multicolumn{2}{|c|}{$\begin{array}{l}\text { ND-Cont. } \\
\text { (eight rats) }\end{array}$} & \multicolumn{2}{|c|}{$\begin{array}{l}\text { HFD-Cont. } \\
\text { (eight rats) }\end{array}$} \\
\hline & Mean & SE & Mean & SE \\
\hline Final body wt (g/rat) & $482 \cdot 8$ & $17 \cdot 9$ & $548 \cdot 6^{\star *}$ & $16 \cdot 8$ \\
\hline Food intake (g/d per rat) & $38 \cdot 3$ & $3 \cdot 6$ & $25 \cdot 3^{*}$ & $3 \cdot 0$ \\
\hline \multicolumn{5}{|l|}{ Wt of WAT (g/rat) } \\
\hline Perirenal & $3 \cdot 2$ & 0.3 & $9 \cdot 9^{\star * \star}$ & 0.8 \\
\hline Epididymal & $5 \cdot 3$ & 0.3 & $18 \cdot 3^{\star \star \star}$ & 0.7 \\
\hline Glucose (mg/l) & 1398 & 42 & 1520 & 57 \\
\hline Insulin (ng/ml) & 2.9 & 0.4 & $6 \cdot 6^{\star \star \star}$ & 0.5 \\
\hline TAG (mg/l) & 1076 & 132 & 2100 & 532 \\
\hline $\operatorname{NEFA}(\mu \mathrm{Eq} / \mathrm{l})$ & 561.4 & $44 \cdot 7$ & $610 \cdot 4$ & $78 \cdot 8$ \\
\hline Total cholesterol (mg/l) & 907 & 36 & $780^{\star}$ & 27 \\
\hline Adiponectin $(\mu \mathrm{g} / \mathrm{l})$ & 39 & 6 & 27 & 3 \\
\hline TNF- $\alpha(p g / m l)$ & $376 \cdot 5$ & $45 \cdot 8$ & $178 \cdot 5^{\star \star}$ & $22 \cdot 6$ \\
\hline Resistin (ng/ml) & $136 \cdot 3$ & $12 \cdot 1$ & $187 \cdot 6^{\star}$ & $15 \cdot 9$ \\
\hline Leptin (ng/ml) & $4 \cdot 2$ & 0.8 & $6 \cdot 8^{\star \star}$ & 0.4 \\
\hline
\end{tabular}

WAT, white adipose tissue.

Mean values were significantly different when compared to that of ND-Cont. group: ${ }^{\star} P<0.05,{ }^{\star \star} P<0.01,{ }^{\star \star \star} P<0.001$ (Student's $t$ test) 
Table 2. Effects of 90-day administration of Eucommia leaf extract (ELE) and Eucommia green leaf powder (EGLP) on body weight, white adipose tissue (WAT) weight and plasma indices in normal diet (ND)-fed rats

(Mean values with their standard errors)

\begin{tabular}{|c|c|c|c|c|c|c|c|c|c|c|}
\hline & & & \multicolumn{4}{|c|}{ ND-ELE } & \multicolumn{4}{|c|}{ ND-EGLP } \\
\hline & \multicolumn{2}{|c|}{ ND-Cont. } & \multicolumn{2}{|c|}{$3 \%$ (eight rats) } & \multicolumn{2}{|c|}{$9 \%$ (eight rats) } & \multicolumn{2}{|c|}{$3 \%$ (eight rats) } & \multicolumn{2}{|c|}{$9 \%$ (eight rats) } \\
\hline & Mean & SE & Mean & SE & Mean & SE & Mean & SE & Mean & SE \\
\hline Final body wt (g/rat) & 482.9 & $17 \cdot 9$ & $462 \cdot 4$ & 18.4 & $426 \cdot 4$ & $24 \cdot 3$ & $459 \cdot 2$ & $25 \cdot 4$ & 423.6 & $26 \cdot 4$ \\
\hline $\begin{array}{l}\text { Food intake (g/d per rat) } \\
\text { Wt of WAT ( } / \text { rat) }\end{array}$ & $38 \cdot 3$ & $3 \cdot 6$ & $38 \cdot 0$ & $5 \cdot 6$ & 41.4 & 4.5 & $37 \cdot 3$ & 4.5 & $42 \cdot 0$ & $6 \cdot 7$ \\
\hline Perirenal & $3 \cdot 2$ & 0.3 & $2 \cdot 6$ & 0.3 & $2 \cdot 3$ & 0.3 & $2 \cdot 7$ & 0.3 & $2 \cdot 3$ & 0.3 \\
\hline Epididymal & $5 \cdot 3$ & 0.3 & 4.7 & 0.4 & 4.3 & 0.3 & 4.8 & 0.3 & 4.3 & 0.3 \\
\hline Glucose (mg/l) & 1398 & 42 & 1344 & 38 & 1395 & 52 & 1346 & 46 & 1111††‡ & 51 \\
\hline Insulin (ng/ml) & 2.9 & 0.4 & $2 \cdot 3$ & 0.3 & $1.9^{\star \star}$ & 0.1 & $2 \cdot 2 \dagger \dagger$ & 0.4 & 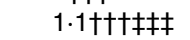 & 0.1 \\
\hline TAG (mg/l) & 1076 & 132 & $795^{\star}$ & 27 & $566^{\star \star \star}$ & 49 & $533+\dagger+$ & 10 & $375+\dagger † \ddagger$ & 8 \\
\hline NEFA $(\mu \mathrm{Eq} / \mathrm{l})$ & 561.4 & 44.7 & $604 \cdot 9$ & $42 \cdot 7$ & $262 \cdot 5^{\star \star \star+++}$ & $21 \cdot 3$ & $353.1+\dagger$ & 33.8 & $574.5 \ddagger \ddagger$ & $27 \cdot 3$ \\
\hline Total cholesterol $(\mathrm{mg} / \mathrm{l})$ & 907 & 36 & $626^{\star \star \star}$ & 10 & $976^{\star \star \star}$ & 40 & $721+\dagger \dagger$ & 7 & $659+\dagger \dagger$ & 32 \\
\hline Adiponectin $(\mu \mathrm{g} / \mathrm{l})$ & 40 & 6 & 42 & 4 & $56^{*}$ & 3 & 38 & 3 & $47 \dagger \dagger$ & 4 \\
\hline TNF- $\alpha(\mathrm{pg} / \mathrm{ml})$ & 376.5 & $45 \cdot 8$ & $294 \cdot 3$ & 24.4 & $219 \cdot 3^{*}$ & $7 \cdot 4$ & 315.4 & $26 \cdot 7$ & $199 \cdot 8+\dagger$ & $20 \cdot 8$ \\
\hline Resistin (ng/ml) & $136 \cdot 3$ & $12 \cdot 1$ & $128 \cdot 3$ & $5 \cdot 6$ & $122 \cdot 9$ & 8.7 & 123.4 & $5 \cdot 1$ & $107 \cdot 9 \dagger$ & $4 \cdot 1$ \\
\hline Leptin ( $\mathrm{ng} / \mathrm{ml})$ & $4 \cdot 2$ & 0.8 & 3.3 & 0.4 & $3 \cdot 2$ & 0.1 & $2 \cdot 8$ & 0.4 & $1.6+\dagger$ & 0.2 \\
\hline
\end{tabular}

HSD, honestly significant difference.

Mean values were significantly different when compared to that of ND-Cont. group: ${ }^{\star} P<0.05,{ }^{\star \star} P<0.01,{ }^{\star \star \star} P<0.001$.

Mean values were significantly different when compared to that of ND-3\%ELE group: $+++P<0.001$ (Tukey HSD).

Mean values were significantly different when compared to that of ND-Cont. group: $\dagger P<0.05, \dagger \dagger P<0.01, \dagger \dagger \dagger P<0.001$.

Mean values were significantly different when compared to that of ND-3\%EGLP group: $¥ P<0.05, \ddagger \ddagger P<0.01, \neq \neq \ddagger P<0.001$ (Tukey’s HSD)

HFD-9\%ELE, HFD-3\%EGLP or HFD-9\%EGLP) for a long period of time exhibited significant, dose-dependent decreases in body weight compared with the HFD-Cont. group $(P<0 \cdot 05$, Table 3$)$.

Administration of ELE and EGLP for 3 months did not significantly alter the food intake by rats fed a ND (Table 2), while chronic administration of $9 \%$ of either form of Eucommia leaves clearly inhibited the intake of food by rats fed a HFD (each $P<0 \cdot 05$, Table 3 ).

Compared with the ND-Cont. groups, the weight of neither perirenal nor epididymal WAT exhibited significant change in 3 months in any ND-ELE or ND-EGLP group (each $P<0 \cdot 1$, Table 2). However, a marked decrease in visceral fat (perirenal or epididymal WAT) was observed in the HFD-3\%ELE and HFD-9\%ELE groups compared with the HFD-Cont. group (each $P<0.001$ ), and this decrease was dose dependent $(P<0 \cdot 001$, Table 3$)$. Similarly, visceral fat weight decreased in a dose-dependent fashion in the HFD-EGLP groups under chronic administration $(P<0.01$ or $P<0.001$, Table 3$)$. The inhibitory effect on the weight of epididymal WAT followed the same pattern as that on perirenal WAT. There were no significant differences in soleus muscle weight among the groups $P<0 \cdot 1$, data not shown).

\section{Plasma levels of lipids, glucose and insulin with chronic} administration of Eucommia leaf extract and Eucommia green leaf powder

Plasma TAG and total cholesterol levels significantly decreased in all the groups in 3 months with ND feeding $(P<0.05$ or 0.001$)$. Change in total cholesterol level was not dose dependent $(P<0 \cdot 1)$, unlike that in plasma TAG level $(P<0 \cdot 05$, Table 2$)$. The ND-9\%ELE and ND-3\%EGLP groups also exhibited marked reductions of plasma levels of NEFA with chronic administration $(P<0.001$ and 0.01 , respectively, Table 2$)$. Under HFD conditions, compared with the Cont. group, the $9 \%$ ELE and $9 \%$ EGLP groups exhibited a significant decrease in plasma TAG levels with chronic administration $(P<0.01$ and $0 \cdot 05$, respectively), and these changes were dose dependent (each $P<0 \cdot 01$, Table 3). Plasma NEFA levels in all the groups decreased slightly with chronic administration, though not to significant extents (each $P<0 \cdot 1$ ), and total cholesterol levels decreased significantly in the HFD-3\%ELE group $(P<0.01)$; however, these effects were not observed for other conditions for administration (Table 3).

A significant decrease in plasma glucose levels was observed in the ND-9\%EGLP group compared with the NDCont. group in 3 months $(P<0 \cdot 01)$, while no difference in this parameter was observed between the ND-ELE group and ND-Cont. groups (both $P<0 \cdot 1$, Table 2). Under HFD conditions, chronic administration of 3 or $9 \%$ ELE and $9 \%$ EGLP yielded significant decreases in plasma glucose level (each $P<0.05$, Table 3).

Administration of $9 \%$ ELE and $3 \%$ or $9 \%$ EGLP yielded marked decreases in plasma insulin levels under ND conditions $(P<0.05,0.05$ and 0.001 , respectively, Table 2$)$, and significantly decreased levels were also observed in the ELE- and EGLP-administered rats under HFD conditions $(P<0.01$ or 0.001 , Table 3$)$. The decreases in levels were dose dependent $(P<0.01$ and $0 \cdot 001$, respectively).

\section{Plasma adipocytokine levels with chronic administration of Eucommia leaf extract and Eucommia green leaf powder}

Under ND conditions, significant increases in plasma adiponectin levels were observed in the ND-9\%ELE and ND-EGLP groups in 3 months compared with the ND-Cont. group (each $P<0.05$ ), with a tendency towards dose dependency (each $P<0 \cdot 1$, Table 2). In the HFD-9\%ELE group, 
Table 3. Effects of 90-day administration of Eucommia leaf extract (ELE) and Eucommia green leaf powder (EGLP) on body weight, white adipose tissue (WAT) weight and plasma indices in high-fat diet (HFD)-fed rats

(Mean values with their standard errors)

\begin{tabular}{|c|c|c|c|c|c|c|c|c|c|c|}
\hline \multirow[b]{3}{*}{ Item } & & & \multicolumn{4}{|c|}{ HFD-ELE } & \multicolumn{4}{|c|}{ HFD-EGLP } \\
\hline & \multicolumn{2}{|c|}{ HFD-Cont. } & \multicolumn{2}{|c|}{$3 \%$ (eight rats) } & \multicolumn{2}{|c|}{$9 \%$ (eight rats) } & \multicolumn{2}{|c|}{$3 \%$ (eight rats) } & \multicolumn{2}{|c|}{$9 \%$ (eight rats) } \\
\hline & Mean & SE & Mean & SE & Mean & SE & Mean & SE & Mean & SE \\
\hline Final body wt (g/rat) & 548.6 & $16 \cdot 8$ & $485 \cdot 3^{*}$ & $13 \cdot 6$ & $422 \cdot 2^{*}$ & $17 \cdot 7$ & $472 \cdot 2 \dagger$ & 13.7 & 427·3†‡ & $12 \cdot 4$ \\
\hline Food intake (g/day/rat) & $25 \cdot 3$ & 3.0 & $19 \cdot 7$ & 3.6 & $15 \cdot 2^{*}$ & 1.7 & $18 \cdot 6$ & 1.5 & $15 \cdot 1 \dagger$ & 1.0 \\
\hline \multicolumn{11}{|l|}{ Wt of WAT (g/rat) } \\
\hline Perirenal & $10 \cdot 0$ & $0 \cdot 8$ & $5 \cdot 6^{\star \star *}$ & 0.4 & $3 \cdot 5^{\star \star \star}$ & 0.6 & $5 \cdot 1 \dagger \dagger$ & 0.5 & $3.3+†$ & 0.5 \\
\hline Epididymal & $18 \cdot 3$ & 0.7 & $13 \cdot 9^{\star \star \star}$ & 0.6 & $5 \cdot 8^{* \star *+++}$ & 0.4 & 7.9†t† & 0.4 & 5.6†††‡ & 0.4 \\
\hline Glucose (mg/l) & 1520 & 17 & $1458^{*}$ & 5 & $1433^{*}$ & 17 & 1469 & 31 & $1458 \dagger$ & 17 \\
\hline Insulin (ng/ml) & $6 \cdot 6$ & 0.5 & $4 \cdot 2^{\star *}$ & 0.5 & $2 \cdot 4^{\star \star \star++}$ & 0.3 & $4 \cdot 2 \dagger \dagger \dagger$ & 0.3 & 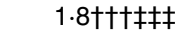 & 0.2 \\
\hline TAG (mg/l) & 2100 & 532 & 1101 & 150 & $605^{\star \star++}$ & 41 & 1169 & 15 & $631 † \ddagger \ddagger$ & 10 \\
\hline NEFA $(\mu \mathrm{Eq} / \mathrm{l})$ & $610 \cdot 4$ & $78 \cdot 8$ & $450 \cdot 8$ & $33 \cdot 8$ & 493.4 & $26 \cdot 2$ & $478 \cdot 0$ & $21 \cdot 6$ & $484 \cdot 3$ & 30.9 \\
\hline Total cholesterol $(\mathrm{mg} / \mathrm{l})$ & 780 & 27 & $655^{\star \star}$ & 28 & 725 & 15 & 758 & 26 & 826 & 8 \\
\hline Adiponectin $(\mu \mathrm{g} / \mathrm{l})$ & 27 & 3 & 42 & 4 & $53^{\star \star}$ & 4 & 39 & 3 & $48 \dagger †$ & 5 \\
\hline TNF- $\alpha(\mathrm{pg} / \mathrm{ml})$ & 178.5 & $22 \cdot 6$ & $137 \cdot 1$ & $15 \cdot 1$ & $63 \cdot 5^{\star+++}$ & $8 \cdot 3$ & 147.5 & $16 \cdot 9$ & 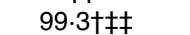 & 6.4 \\
\hline Resistin (ng/ml) & 187.6 & $15 \cdot 9$ & $175 \cdot 9$ & $15 \cdot 9$ & $111 \cdot 4^{\star \star++}$ & $11 \cdot 0$ & 184.1 & $16 \cdot 9$ & 117.0††キキ & 7.4 \\
\hline Leptin (ng/ml) & $6 \cdot 8$ & 0.4 & 5.9 & 0.7 & $6 \cdot 7$ & 0.8 & $6 \cdot 0$ & 0.5 & $7 \cdot 6$ & 0.6 \\
\hline
\end{tabular}

Cont., control; HSD, honestly significant difference.

Mean values were significantly different when compared to that of HFD-Cont. group: ${ }^{\star} P<0.05,{ }^{\star \star} P<0.01,{ }^{\star \star \star} P<0.001$.

Mean values were significantly different when compared to that of HFD-3\%ELE: $++P<0.01,+++P<0.001$ (Tukey HSD).

Mean values were significantly different when compared to that of HFD-Cont. group: $\uparrow P<0.05, \emptyset \dagger P<0.01, \dagger \dagger \dagger P<0.001$.

Mean values were significantly different when compared to that of HFD-3\%EGLP group: $¥ P<0.05, \ddagger \ddagger P<0.01, \neq \ddagger \ddagger P<0.001$ (Tukey's HSD).

significantly increased levels of plasma adiponectin were observed and increases were observed in the HFD-3\%ELE and HFD-3\%EGLP groups at similar concentrations (each $P<0 \cdot 01$, Table 3 ). The strength of effect in the HFD-EGLP groups was slightly weaker than that in the HFD-ELE groups.

When plasma TNF- $\alpha$ levels were examined in the ND-Cont., ND-ELE and ND-EGLP groups, a significant decrease was observed in the ND-9\%ELE and ND-9\%EGLP groups under chronic administration conditions $(P<0.05$ and 0.01 , respectively, Table 2). However, plasma resistin and leptin levels tended to decrease in the ND-ELE groups, though without significant dose dependency $(P<0 \cdot 1$, Table 2$)$. On the other hand, significant decreases in plasma resistin and leptin levels were observed in the chronic, high-dose EGLP groups under ND conditions $(P<0.05$ and 0.01 , respectively, Table 2$)$. Similarly, significant decrease in plasma TNF- $\alpha$ levels were observed in the chronic HFD-9\%ELE and HFD-9\%EGLP administration groups (both $P<0.05$, Table 3 ), and these decreases were dose dependent $(P<0.001)$. Plasma resistin levels dose dependently decreased with chronic administration of ELE and EGLP under HFD conditions (both $P<0.01$ ), and significantly decreased levels were observed in the HFD9\%ELE and HFD-9\%EGLP groups (both $P<0.01$, Table 3). No significant change in plasma leptin levels was observed even in the chronic high-dose administration groups under HFD conditions $(P<0 \cdot 1$, Table 3$)$.

Gene expression related to glucose metabolism with chronic administration of Eucommia leaf extract and Eucommia green leaf powder

In the liver, no significant increase in the expression of glucose metabolic genes was observed in the ND-ELE or ND-EGLP groups (both $P<0 \cdot 1$, supplementary Table S2 (available online only at http://journals.cambridge.org), Fig. 1). Both the groups exhibited increased levels of isocitrate dehydrogenase 3 $\left(\mathrm{NAD}^{+}\right) \alpha$ and NADH dehydrogenase flavoprotein 1 mRNA in the perirenal WAT $(P<0.05$ or 0.001$)$; the increase in the NDEGLP group was higher than that in the ND-ELE group, though not to a significant extent $(P<0 \cdot 1$, supplementary Table S2 (available online only at http://journals.cambridge.org), Fig. 1). The ND-9\%EGLP group exhibited induction of mRNA expression of dihydrolipoamide succinyltransferase in the perirenal WAT. ATP synthase, $\mathrm{H}^{+}$transporter, mitochondrial F1 complex and delta subunit mRNA levels were increased in the perirenal WAT of the ND-EGLP group (each $P<0 \cdot 001$ ). The levels of solute carrier family 2 (facilitated GLUT), member 4 (GLUT4), pyruvate kinase, citrate synthase and NADH dehydrogenase flavoprotein $1 \mathrm{mRNA}$ in the soleus muscle were significantly increased after chronic administration of ELE and EGLP $(P<0 \cdot 05$, supplementary Table S2 (available online only at http://journals.cambridge.org), Fig. 1). The ND-EGLP group exhibited increase in isocitrate dehydrogenase $3\left(\mathrm{NAD}^{+}\right)$ $\alpha$ mRNA levels in the soleus muscle $(P<0.05$, supplementary Table S2 (available online only at http://journals.cambridge. org), Fig. 1).

Under HFD conditions, chronic administration of ELE and EGLP induced mRNA expression of glucokinase, citrate synthase and NADH dehydrogenase flavoprotein 1 in rat liver $(P<0.05$, Table 4 , Fig. 1$)$. In the perirenal WAT of HFD-fed rats, levels of isocitrate dehydrogenase $3\left(\mathrm{NAD}^{+}\right)$ $\alpha$, dihydrolipoamide succinyltransferase, NADH dehydrogenase flavoprotein 1 and ATP synthase, $\mathrm{H}^{+}$transporting, mitochondrial F1 complex, delta subunit mRNA were markedly decreased by the chronic administration of ELE and EGLP $(P<0.05$ or 0.001$)$. In contrast to the strongly decreased levels of perirenal WAT GLUT4 mRNA in rats fed EGLP $(P<0.001)$, administration of the ELE slightly decreased 


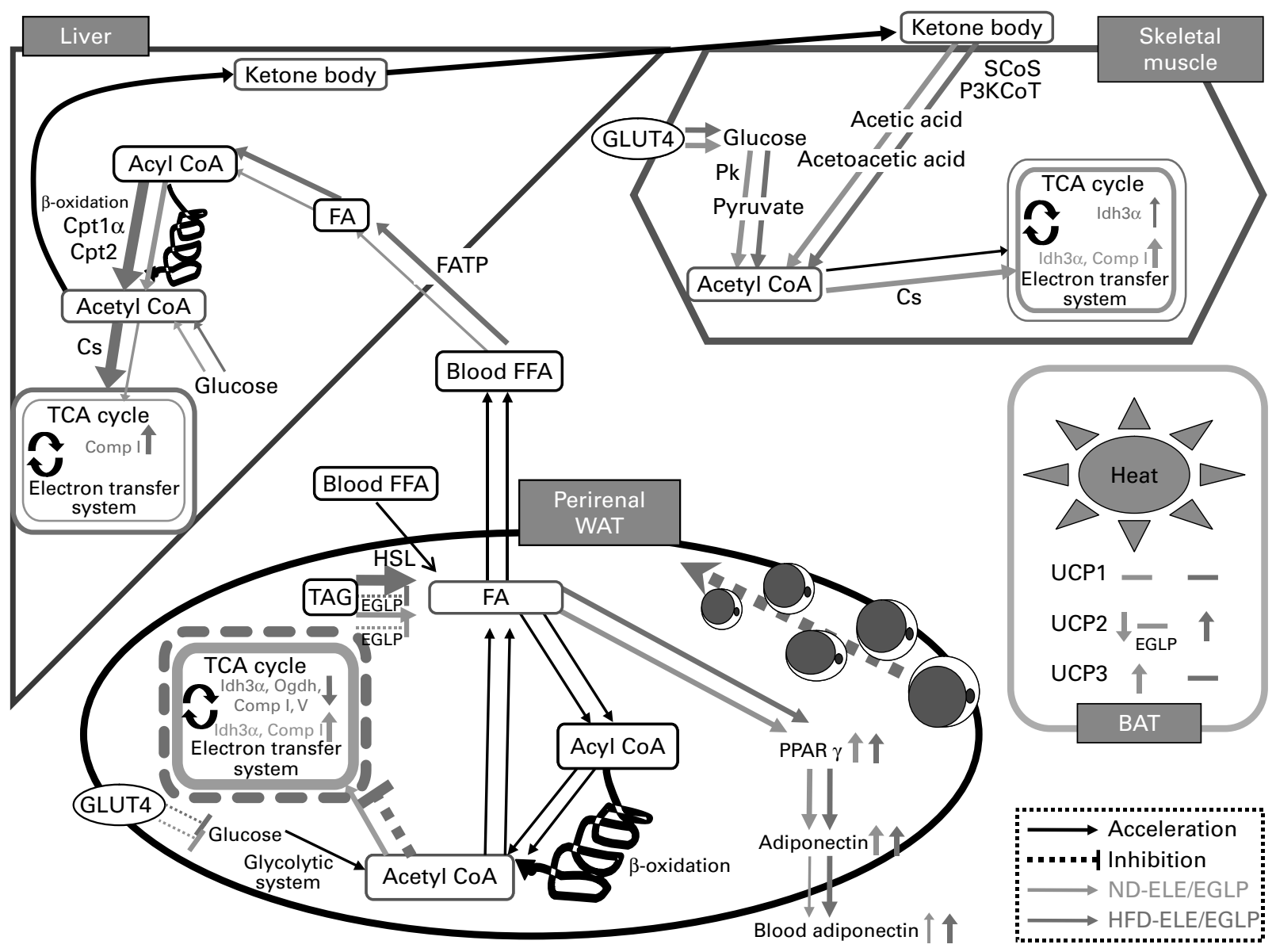

Fig. 1. Diagrammatic illustration of gene expression related to metabolism affected by chronic administration of Eucommia leaves in rats fed a normal diet (ND) or high-fat diet (HFD). Solid arrow: acceleration of metabolic function in Eucommia leaf extract (ELE)/Eucommia green leaf powder (EGLP)-fed rats under ND or HFD conditions. Dash line: inhibition of metabolic function in ELE/EGLP-fed rats under ND or HFD conditions. Green line: ND conditions, pink line: HFD conditions. TCA cycle box indicated by a green/pink solid line: activation of TCA cycle and/or electron transfer system. Tricarboxylic acid (TCA) cycle box indicated by a pink dash line: inhibition of TCA cycle and electron transfer system. Up-pointing green/pink arrow indicated in TCA cycle box: increase in gene expressions under ND/HFD conditions. Down-pointing pink arrow in TCA cycle box: decrease in gene expressions under HFD conditions. FA, fatty acid; FFA, free FA; SCoS, succinyl-CoA synthetase; P3KCoT, peroxisomal 3-ketoacyl-CoA thiolase; Cpt1 $\alpha$, carnitine palmitoyltransferase 1 $\alpha$; Cpt2, carnitine palmitoyltransferase 2; Pk, pyruvate kinase; Cs, citrate synthase; Idh3 $\alpha$, isocitrate dehydrogenase $3\left(\mathrm{NAD}^{+}\right) \alpha$; Comp I, NADH dehydrogenase flavoprotein 1; Comp V, ATP synthase, $\mathrm{H}^{+}$transporting, mitochondrial F1 complex, delta subunit; Ogdh, dihydrolipoamide succinyltransferase; $\mathrm{HSL}$, hormone-sensitive lipase; UCP, uncoupling protein (ATP synthase); FATP, fatty acid transport protein; WAT, white adipose tissue; BAT, brown adipose tissue; HSL, hormone-sensitive lipase; FATP, FA transport protein.

GLUT4 mRNA level $(P<0 \cdot 05$, Table 4, Fig. 1). In the soleus muscle of rats under HFD conditions, $9 \%$ ELE significantly increased the levels of GLUT4 and pyruvate kinase mRNA (both $P<0.05$ ), while 9\% EGLP markedly increased GLUT4 and isocitrate dehydrogenase $3\left(\mathrm{NAD}^{+}\right) \alpha$ mRNA levels (both $P<0 \cdot 05$, Table 4, Fig. 1).

Gene expression related to lipid metabolism with chronic administration of Eucommia leaf extract and Eucommia green leaf powder

ND-ELE and ND-EGLP groups exhibited increase in liver carnitine palmitoyltransferase $1 \alpha$ and carnitine palmitoyltransferase 2 mRNA levels, and these effects were dose dependent $(P<0.05$ or 0.001 , supplementary Table S2 (available online only at http://journals.cambridge.org), Fig. 1). The increases in mRNA level were higher in the ND-EGLP group than in the ND-ELE group. Only the ND-9\%EGLP group exhibited induction of fatty acid transport protein mRNA expressions in the liver $(P<0 \cdot 05)$. Chronic administration clearly decreased hormone-sensitive lipase mRNA levels in perirenal WAT of ND-3\%ELE and ND-9\%ELE groups $(P<0.05)$, while the ND-9\%EGLP group exhibited significant increase in hormonesensitive lipase mRNA levels $(P<0.05$, supplementary Table S2 (available online only at http://journals.cambridge.org), Fig. 1).

In rat liver under HFD conditions, levels of fatty acid transport protein, carnitine palmitoyltransferase 1 and carnitine palmitoyltransferase 2 mRNA were markedly increased by chronic administration of ELE and EGLP $(P<0.05$ or 0.001 , Table 4, Fig. 1). The HFD-ELE groups exhibited significant increase in hormone-sensitive lipase mRNA levels in perirenal WAT $(P<0 \cdot 001)$, while the HFD-EGLP groups exhibited significant decrease in hormone-sensitive lipase mRNA level $(P<0 \cdot 05$, Table 4, Fig. 1).

Gene expression related to ketone body metabolism with chronic administration of Eucommia leaf extract and Eucommia green leaf powder

Chronic administration of ELE led to the increase in succinylCoA synthetase and peroxisomal 3-ketoacyl-CoA thiolase 
Table 4. Gene expression analysis by real-time PCR in liver, white adipose tissue (WAT), muscle and brown adipose tissue (BAT) after 90-d administration of Eucommia leaf extract (ELE) and Eucommia green leaf powder (EGLP) under high-fat diet (HFD)

(Mean values with their standard errors)

\begin{tabular}{|c|c|c|c|c|c|c|c|c|c|}
\hline \multirow[b]{3}{*}{ Gene name } & \multirow[b]{3}{*}{ Accession number } & \multicolumn{4}{|c|}{ HFD-ELE (fold of cont.) } & \multicolumn{4}{|c|}{ HFD-EGLP (fold of cont.) } \\
\hline & & \multicolumn{2}{|c|}{$3 \%$ (eight rats) } & \multicolumn{2}{|c|}{$9 \%$ (eight rats) } & \multicolumn{2}{|c|}{$3 \%$ (eight rats) } & \multicolumn{2}{|c|}{$9 \%$ (eight rats) } \\
\hline & & Mean & SE & Mean & SE & Mean & SE & Mean & SE \\
\hline \multicolumn{10}{|l|}{ Liver } \\
\hline Gck & NM 012565 & $1 \cdot 25^{\star}$ & 0.07 & $2 \cdot 16^{\star+}$ & 0.12 & 1.21 & 0.13 & $1.50 \dagger$ & 0.18 \\
\hline Cs & NM 130755 & $1.89^{\star}$ & 0.21 & $3 \cdot 15^{\star+}$ & 0.62 & $1.89 \dagger$ & 0.29 & $2.44 \dagger$ & 0.45 \\
\hline Comp I & CB5449004 & $1 \cdot 21$ & $0 \cdot 10$ & $1.54^{\star+}$ & $0 \cdot 18$ & $1 \cdot 38$ & 0.12 & $1.77 \dagger$ & 0.28 \\
\hline FATP & NM 053580 & 1.26 & 0.09 & $1.66^{\star+}$ & 0.23 & 1.33 & 0.10 & $1.65 \dagger$ & 0.21 \\
\hline Cpt1 $\alpha$ & NM 031559 & $2 \cdot 08^{\star}$ & 0.34 & $2 \cdot 94^{\star+}$ & 0.41 & 1.29††† & 0.17 & $1.42 \dagger \ddagger$ & 0.14 \\
\hline Cpt2 & NM 012930 & $2 \cdot 11^{*}$ & 0.11 & $2 \cdot 72^{*+}$ & 0.28 & $2.18 \dagger$ & 0.29 & $2.70 \dagger$ & 0.36 \\
\hline \multicolumn{10}{|l|}{ WAT (perirenal) } \\
\hline GLUT4 & NM 012751 & $0.88^{*}$ & 0.27 & $0.70^{\star+}$ & 0.18 & 0.19††† & 0.06 & $0.11 \dagger \dagger \dagger$ & 0.12 \\
\hline $\operatorname{Idh} 3 \alpha$ & NM 053638 & $0 \cdot 10^{\star \star \star}$ & 0.08 & $0.07^{\star \star \star+}$ & 0.08 & $0.38+\dagger \dagger$ & 0.29 & $0.24+\dagger \dagger$ & 0.19 \\
\hline Ogdh & Al412142 & $0.38^{\star \star \star}$ & $0 \cdot 16$ & $0.22^{\star \star \star}$ & 0.13 & $0.57 \dagger$ & 0.21 & $0.41 \dagger+\dagger$ & 0.18 \\
\hline Comp I & CB5449004 & $0.37^{\star \star \star}$ & 0.19 & $0 \cdot 10^{\star \star \star}$ & 0.09 & $0.96 \dagger+\dagger$ & 0.23 & $0.51 \dagger$ & 0.17 \\
\hline Comp V & NM 139106 & $0 \cdot 18^{\star \star \star}$ & 0.31 & $0.26^{\star \star \star}$ & 0.23 & $0.87 \dagger \dagger \dagger$ & 0.21 & $0.55 \dagger \ddagger$ & 0.16 \\
\hline $\mathrm{HSL}$ & NM 012859 & $3.52^{\star \star *}$ & 0.21 & $6 \cdot 04^{\star \star *+}$ & 0.37 & 0.98 & 0.17 & $0.66 \dagger$ & 0.10 \\
\hline PPAR $\gamma$ & NM 013124 & 1.14 & 0.13 & $1.43^{*}$ & $0 \cdot 11$ & $1 \cdot 18$ & 0.16 & 1.33 & 0.17 \\
\hline Adiponectin & NM 144744 & $1.41^{*}$ & 0.12 & $2 \cdot 20^{*+}$ & 0.23 & 1.34 & $0 \cdot 21$ & $1.89 \dagger$ & 0.35 \\
\hline \multicolumn{10}{|l|}{ Soleus muscle } \\
\hline GLUT4 & NM 012751 & $1 \cdot 16$ & 0.18 & $1.31^{*}$ & $0 \cdot 10$ & $1 \cdot 17$ & 0.13 & $1.59 \dagger$ & 0.19 \\
\hline $\mathrm{Pk}$ & NM 053297 & $1.48^{*}$ & 0.17 & $2 \cdot 05^{\star+}$ & 0.28 & 1.09 & 0.11 & 1.36 & 0.13 \\
\hline $\operatorname{Idh} 3 \alpha$ & NM 053638 & 0.96 & 0.29 & 1.01 & 0.27 & 1.38 & 0.25 & $1.60 \dagger$ & 0.21 \\
\hline Cs & NM 130755 & 0.90 & 0.23 & 1.09 & 0.22 & $1 \cdot 14$ & 0.23 & 1.09 & 0.18 \\
\hline Comp I & CB5449004 & 1.02 & 0.17 & 1.15 & $0 \cdot 11$ & 1.26 & 0.13 & 1.30 & 0.12 \\
\hline SCoS & J03621 & $1.57^{\star}$ & 0.18 & $1.88^{\star}$ & 0.20 & $1 \cdot 18$ & 0.13 & 1.20 & 0.19 \\
\hline P3КСоT & J02749 & 1.08 & $0 \cdot 10$ & 1.34 & 0.11 & $1.49 \dagger$ & 0.15 & $1.65 \dagger$ & 0.12 \\
\hline \multicolumn{10}{|l|}{ BAT } \\
\hline UCP2 & NM 019354 & 1.01 & 0.12 & $1.49^{*+}$ & 0.18 & $1 \cdot 11$ & 0.28 & $1.45 \dagger$ & 0.25 \\
\hline UCP3 & NM 013167 & 0.88 & 0.43 & 1.13 & 0.31 & 0.86 & 0.49 & 1.09 & 0.38 \\
\hline
\end{tabular}

cont., control; Gck, glucokinase; Cs, citrate synthase; Comp I, NADH dehydrogenase flavoprotein 1; FATP, fatty acid transport protein; Cpt1 $\alpha$, carnitine palmitoyltransferase $1 \alpha$ Cpt2, carnitine palmitoyltransferase 2 ; Idh $3 \alpha$, isocitrate dehydrogenase 3 (NAD $\left.{ }^{+}\right) \alpha$; Ogdh, dihydrolipoamide succinyltransferase; Comp V, ATP synthase, $\mathrm{H}^{+}$transporting, mitochondrial F1 complex, delta subunit; HSL, hormone-sensitive lipase; Pk, pyruvate kinase; SCoS, succinyl-CoA synthetase; P3KCoT, peroxisomal 3-ketoacyl-CoA thiolase; UCP, uncoupling protein (ATP synthase); HSD, honestly significant difference.

Mean values were significantly different when compared to that of HFD-Cont. group: ${ }^{\star} P<0.05,{ }^{\star *} P<0.01,{ }^{\star * \star} P<0.001$.

Mean values were significantly different when compared to that of HFD-3\%ELE group: $+P<0.05$ (Tukey HSD).

Mean values were significantly different when compared to that of HFD-Cont. group: $\dagger P<0.05, \dagger \dagger P<0.01, \dagger \dagger \dagger P<0.001$

Mean values were significantly different when compared to that of HFD-3\%EGLP group: $\ddagger P<0.05, \ddagger \ddagger P<0.01$, 㧊 $P<0.001$ (Tukey’s HSD).

mRNA levels in the soleus muscle of rats under ND conditions $(P<0 \cdot 05)$, while EGLP administration significantly increased only the level of succinyl-CoA synthetase mRNA $(P<0 \cdot 05$, supplementary Table S2 (available online only at http:// journals.cambridge.org), Fig. 1).

In the soleus muscle of HFD-fed rats, ELE led to a remarkable increase in the level of succinyl-CoA synthetase mRNA $(P<0.05)$, while EGLP significantly increased peroxisomal 3-ketoacyl-CoA thiolase mRNA level $(P<0 \cdot 05$, Table 4, Fig. 1).

PPAR $\gamma$ and adiponectin gene expression with chronic administration of Eucommia leaf extract and Eucommia green leaf powder

In the perirenal WAT, chronic administration of 9\% ELE and 9\% EGLP led to the increase in PPAR $\gamma$ mRNA levels $(P<0.05$ and 0.001 , respectively), and the expression of this gene was higher in the EGLP group than in the ELE group under ND conditions (supplementary Table S2 (available online only at http://journals.cambridge.org), Fig. 1). Although no differences between the groups were observed in increase in adiponectin mRNA level by the ELE and EGLP, 9\% ELE and 9\% EGLP each yielded significant increase in adiponectin mRNA level $(P<0 \cdot 05$, supplementary Table S2 (available online only at http://journals.cambridge.org), Fig. 1).

In the perirenal WAT of HFD-fed rats, ELE led to significant increases in PPAR $\gamma$ and adiponectin mRNA levels (both $P<0.05$ ), while EGLP increased the level of adiponectin mRNA $(P<0.05)$ but not that of PPAR $\gamma$ mRNA $(P<0 \cdot 1$, Table 4, Fig. 1).

Uncoupling protein gene expression with chronic administration of Eucommia leaf extract and Eucommia green leaf powder

In rat BAT under ND conditions, ELE administration led to a marked increase in the level of uncoupling protein (UCP) 3 mRNA $(P<0.05)$, with a trend towards decrease in UCP2 mRNA levels $(P<0.05)$, while EGLP administration did not significantly increased those mRNA levels $(P<0 \cdot 1$, 
supplementary Table S2 (available online only at http:// journals.cambridge.org), Fig. 1).

In BAT of HFD-fed rats, chronic administration of $9 \%$ ELE and $9 \%$ EGLP led to a significant increase in UCP2 mRNA levels $(P<0.05)$ but did not affect the expression of UCP3 mRNA $(P<0 \cdot 1$, Table 4, Fig. 1). Neither ND- nor HFD-fed rats supplemented with ELE or EGLP did exhibit marked change in UCP1 mRNA expression (both $P<0 \cdot 1$, data not shown).

\section{Discussion}

The HFD-Cont. group exhibited primary signs of progressive metabolic syndrome, such as increase in body weight and energy intake with marked accumulation of visceral fat. Changes in blood indices including hyperinsulinaemia and hypertriacylglycerolaemia were observed, as well. Chronic feeding of a HFD decreased plasma adiponectin levels and increased plasma levels of resistin and leptin.

It has been reported that neither a $35 \mathrm{~d}$ administration of ELE at a high dose (3 or $6 \mathrm{~g}$ dried leaves $/ \mathrm{kg}$ rat body weight per d) nor a 10-week administration at a low dose (approximately $15.5 \mathrm{mg} / \mathrm{d}$ in hamsters) led to a significant decrease in the body weight of animals ${ }^{(20,21)}$. High-dose administration of ELE led to a significant decrease in the weight of $\mathrm{WAT}^{(20)}$, while low-dose administration did not affect WAT weight ${ }^{(21)}$. In the present study, 3-month administration of ELE and EGLP controlled body weight gain and WAT weight, and this control was particularly strong in HFD rats. Even in ND rats, body weight and WAT weight each decreased slightly, though not to significant extents. These findings show that Eucommia leaves, regardless of type of processing, cause loss of body and WAT weight, depending on the dose and duration of administration.

Regarding carbohydrate metabolism, rats treated with $3 \%$ EGLP for 3 months exhibited marked changes in plasma glucose levels under both ND and HFD conditions, and marked changes in plasma glucose levels were observed in rats treated with ELE under HFD conditions, but not under ND conditions. Since ELE includes different levels of components than does EGLP, this finding suggests that the differences in component ratios may affect glucose metabolism. We could not clearly determine the hypoglycaemic effects of ELE and EGLP under HFD conditions since plasma glucose levels did not increase enough in the animal model of obesity used for this analysis. However, Lee et al. reported that ELE controlled plasma glucose levels in type 1 diabetes model rats and type 2 diabetes model mice $(\mathrm{C} 57 \mathrm{BL} / \mathrm{KsJ}-\mathrm{db} / \mathrm{db} \text { mice })^{(22,23)}$. In the present study, while chronic feeding of HFD significantly increased plasma insulin levels, the magnitude of increase was markedly decreased by ELE or EGLP administration over 3 months. Decrease in plasma insulin levels in ELE- and EGLPsupplemented rats was also confirmed under ND conditions. The decrease observed in EGLP-fed rats was stronger than that in ELE-fed rats under both the conditions. This suggests that chronic administration of ELE or EGLP may improve insulin resistance in the rat model of HFD-induced obesity.

A real-time PCR study showed that chronic administration of ELE and EGLP to rats fed a ND significantly increased the activity of the glycolytic system, tricarboxylic acid (TCA) cycle and electron transport system in skeletal muscle, and markedly increased the activity of the TCA cycle and the electron transport system in WAT. Chronic administration of ELE and EGLP significantly increased the activity of the glycolytic system in skeletal muscle, and TCA cycle and electron transport system in the liver of rats fed a ND. On the other hand, the activity of the TCA cycle and the electron transport system in WAT pronouncedly decreased under HFD conditions. GLUT4 mRNA expression was increased in skeletal muscle and decreased in WAT under both dietary conditions. This suggests that the effects of ELE/EGLP on visceral fat differ depending upon dietary conditions, and that the ELE- or EGLP-fed rats are no longer able to maintain the shape of adipose cells in which TAG were stored because of decrease in ATP production in WAT cells under HFD conditions. Marked use of glucose in skeletal muscle, which may decrease circulating glucose levels under ND or HFD condition, was enhanced in both the groups of rats.

One study reported that increases in plasma levels of TAG, NEFA and cholesterol were directly related to a higher incidence of obesity and other lipid-associated diseases ${ }^{(24)}$. In the present study, a 3-month administration of 9\% ELE or $9 \%$ EGLP suppressed plasma TAG levels in rats under ND and HFD conditions. A hypolipidaemic effect of ELE has been previously reported ${ }^{(18)}$, in which ELE significantly decreased the magnitude of increase in levels of TAG in plasma that followed a single administration of lipids. In addition, a recent study showed that ELE stimulated liver lipid metabolism and decreased the plasma level of TAG and cholesterol in hamsters ${ }^{(21)}$. The present study demonstrated that inhibitory effects of chronic administration of ELE and EGLP at doses on plasma TAG levels were more marked in HFD-fed rats than in ND-fed rats. Therefore, ELE and EGLP, regardless of their concentrations and terms of administration, might affect control of absorption of TAG derived from foods into the bloodstream and the activation of lipid metabolism, particularly fatty acid $\beta$-oxidation, in the liver. On the one hand, HFD-9\%ELE, HFD-3\%EGLP and HFD-9\%EGLP rats did not exhibit such decreased levels of plasma total cholesterol as in rats supplemented with ELE and EGLP under ND conditions. A slight tendency towards a decrease in plasma fatty acid levels was observed in rats supplemented with ELE and EGLP under HFD conditions, though it was NS. Dose dependency of decrease in the level of plasma cholesterol in ELE- and EGLP-fed rats under ND conditions could not be confirmed. In HFD conditions, only $3 \%$ ELE significantly decreased plasma cholesterol levels. Low-dose administration of ELE, regardless of dietary conditions, clearly decreased plasma cholesterol levels in rats, suggesting that chronic administration of ELE or EGLP may improve hyperlipaemia in rats with HFDinduced obesity.

Real-time PCR analysis showed that chronic administration of ELE and EGLP increased fatty acid $\beta$-oxidation in the liver. In HFD-fed rats, uptake of fatty acids into the liver was increased by ELE and EGLP, followed by an increase in fatty acid $\beta$-oxidation and ATP production, which may decrease circulating NEFA levels. Concomitantly, activation of lipolysis in the WAT of rats fed a HFD supplemented with ELE, but not with EGLP, was confirmed. Chronic administration of ELE and EGLP induced mRNA expression of 
PPAR $\gamma$ and adiponectin, which depends on the accumulation of visceral fat to improve insulin resistance or hyperlipaemia, while it did not affect fatty acid $\beta$-oxidation in WAT of rats under ND or HFD conditions. Indeed, in the present study, chronic administration of ELE and EGLP tended to decrease plasma NEFA levels and significantly increased plasma adiponectin levels under HFD conditions. A recent study reported that ELE suppressed hepatic activities of fatty acid synthase and HMG-CoA reductase, and increased hepatic $\beta$-oxidation activity in HFD-fed hamsters ${ }^{(21)}$, resulting in decreased plasma levels of fatty acids and cholesterol and hepatic levels of cholesterol. Moreover, our findings show that chronic administration of ELE or EGLP causes a simultaneous activation of ketone body's use in skeletal muscle and of hepatic fatty acid $\beta$-oxidation in rats regardless of dietary conditions.

$\mathrm{UCP}^{(25)}$, of which at least three closely related homologues have been cloned, appear to act as fatty acid anion transporters other than transporters of protons ${ }^{(26)}$. UCP1 (thermogenin) is highly expressed in BAT, where it is involved in nonshivering thermogenesis ${ }^{(27)}$. The present study showed that chronic administration of neither ELE nor EGLP affected UCP1 mRNA expression in BAT of rats regardless of dietary conditions, while mRNA levels of UCP2 in BAT were decreased by ELE under ND conditions and increased by ELE and EGLP under HFD conditions. The increase in UCP2 mRNA expressions may act to minimise the generation of mitochondrially derived reactive oxygen species ${ }^{(28)}$. In rat BAT under ND conditions, chronic ELE or EGLP administration enhanced the level of UCP3 mRNA, which is associated with fatty acid oxidation in various tissues ${ }^{(29)}$, while no significant difference was observed under HFD conditions.

Adipocytokines are specifically secreted from visceral fat tissues, and plasma levels of adiponectin, which has an anti-arteriosclerosis effect and improves insulin sensitivity, increase in inverse proportion to increase in the weight of visceral fat tissues. Chronic administration of ELE and EGLP caused significant and dose-dependent increases in plasma adiponectin levels, with clear loss of the visceral fat pad in rats of both the conditions. In addition, this result was supported by a real-time PCR study in which the chronic administration of ELE and EGLP induced the gene expression of PPAR $\gamma$ and adiponectin in the perirenal WAT of rats under both the dietary conditions.

Plasma levels of resistin, which induces insulin resistance, were particularly decreased by chronic administration of EGLP at a high dose under ND conditions. These decreases in plasma resistin levels in rats supplemented with ELE and EGLP were even observed under HFD conditions. It can be inferred that in chronic, high-dose administration, such changes in plasma levels of adipocytokines may lead to improvement of hyperinsulinaemia. It is known that short-term treatment with a HFD induces insulin resistance, though this is unrelated to the plasma levels of TNF- $\alpha$ which is involved in inflammatory reactions ${ }^{(24,30)}$. In the present study, plasma levels of TNF- $\alpha$ were decreased by chronic administration of HFD. Administration of HFD for 1 month significantly increased plasma TNF- $\alpha$ levels compared with ND (data not shown). Although the reason remains unclear, this might show that the endocrine environment in TNF- $\alpha$ secretion from WAT was changed by the administration period of HFD. The chronic, high-dose administration of ELE and EGLP also reduced the magnitude of decrease in plasma TNF- $\alpha$ level compared with that in rats fed a ND or HFD without supplementations. Plasma levels of leptin, which is involved in appetite regulation, were increased by a HFD. Plasma leptin levels decreased after chronic administration of $9 \%$ EGLP under ND conditions, while no conditions except $9 \%$ EGLP significantly decreased plasma leptin level in rats fed either diet.

In conclusion, these findings suggest that chronic administration of Eucommia leaf, regardless of the type of processing used and its concentration, potentially exerts anti-obesity and anti-metabolic syndrome effects, and systematically stimulates metabolic function in rats across several organs, including decrease in ATP production in WAT, acceleration of fatty acid $\beta$-oxidation in the liver and increase in the use of ketone body/glucose in skeletal muscle. The anti-obesity and anti-metabolic syndrome activities of Eucommia leaf in this rat model may be maintained through secretion and regulation of adipocytokines that depend on the accumulation of visceral fat to improve insulin resistance or hyperlipaemia.

\section{Acknowledgements}

The authors are grateful to M. Mikame for technical assistance and S. Nakao for animal care assistance. The study was carried out with support from Kobayashi Pharmaceutical Company, Limited (Osaka, Japan). ELE and EGLP were also provided by Kobayashi Pharmaceutical Company, Limited. The study sponsors had no role in the design and conduct of the study; the collection, management, analysis and interpretation of the data; the preparation, approval and submission of the manuscript.

All the authors contributed to the writing of the manuscript and interpretation of the results, reviewed its content and approved the final version submitted for publication. T. F. was the principal investigator for the design of the study and supervision of data collection; T. H., A. W., N. K., Y. Y., K. F., T. U., H. S. and S. N. contributed to the design of the study, data collection and laboratory analysis; Y. Y. contributed to laboratory analysis.

None of the authors had a financial or personal conflict of interest in relation to the present study.

\section{References}

1. Chinese Materia Medica Dictionary (1977) Jiangsu New Medical College, p. 1031. Shanghai: Shanghai Science and Technology Publishing House.

2. Nakamura T, Nakazawa Y, Onizuka S, et al. (1997) Studies on the constituents of Eucommia ulmoides Iridoisd from the leaves. Nat Med 51, 275-277.

3. Li H, Chen B \& Yao S (2005) Application of ultrasonic technique for extracting chlorogenic acid from Eucommia ulmodies Oliv. Ultrason Sonochem 12, 295-300.

4. Takamura C, Hirata T, Yamaguchi Y, et al. (2007) Studies on the chemical constituents of green leaves of Eucommia ulmoides Oliv. J Nat Med 61, 220-221.

5. Takamura C, Hirata T, Ueda T, et al. (2007) Iridoids from the green leaves of Eucommia ulmoides. J Nat Prod 70, $1312-1316$.

6. Qin ZD, Wu YZ, Yu ZQ, et al. (1997) Studies on the Eucomia cortex and leaves. West-North Univ J 64-71. 
7. Guizhou Province Institute for Drug Control and Guizhou Province Chinese Medicine Research Laboratories (1978) Chin Med Herbs Res 8, 59-63.

8. Guizhou Province Hypotensive Clinical Committee (Institute for Drug Control and Chinese Medicine Research Laboratories) (1978) A clinical study on replacement of Eucommia cortex with Eucommia leaves. New Med J 10, 30-32.

9. Liu D \& Li CW (1980) Clinical evalution of Eucommia cortex and leaves by 102 type of hypertention. Shanxi Chin Med $1,27-30$.

10. Shanxi Province Eucommia Clinical Review Committee (1995) Replacement of Eucommia cortex with Eucommia leaves for hypertension treatment. A clinical review with 621 cases, pp. 19-54. Xian: Shanxi Eucommia Development Company.

11. Namba T, Hattori M, Yie J, et al. (1986) Studies on Tu-Chung leaves (1): pharmacological effects of the water extract in vivo. J Trad Med 3, 89-97.

12. Nomura Y, Kaneko S, Kitamura Y, et al. (1986) Effects of Tu-chung leaves extract on adenylate cyclase of rat brain and heart receptor. J Trad Med 3, 328-329.

13. Nakazawa $\mathrm{Y}$, Odagiru $\mathrm{N}$, Imai $\mathrm{R}$, et al. (1997) Effect of Eucommia leaf exstract on blood pressure (1): effect on blood pressure in spontaneous hypertensive rats (SHR). Nat Med 51, 392-398.

14. Tagawa C, Nakazawa Y, Tagashira E, et al. (2005) Effect of Eucomia leaf (Eucommia ulmoides Oliver; Du-Zhong yge) extract on blood pressure (2). Nat Med 59, 117-120.

15. Ohmori R, Iwamoto $\mathrm{T}$, Tago $\mathrm{M}$, et al. (2005) Antioxidant activity of various teas against free radicals and LDL oxidation. Lipids 40, 849-853.

16. Yang J, Kato K, Noguchi K, et al. (2003) Tochu (Eucommia ulmoides) leaf extract prevents ammonia and vitamin $\mathrm{C}$ deficiency induced gastric mucosal injury. Life Sci 73, 3245-3256.

17. Fujikawa T, Tsuboi T, Kawamura N, et al. (2007) Eucommia ulmoides Oliver as a prophylactic for restraint stress in waterinduced gastric erosion in rats. Int Symp Eucommia ulmoides 1, 67-71.

18. Ando C, Kobayashi T, Tsukamoto S, et al. (2007) Antiobesity effects of Eucommia ulmoides leaves. Int Symp Eucommia ulmoides 1, 63-66.
19. Matsuzawa Y (2005) Adiponectin: identification, physiology and clinical relevance in metabolic and vascular disease. Atheroscler Suppl 6, 7-14.

20. Metori K, Ohashi S, Takahashi S, et al. (1994) Effects of Du-Zhong leaf extract on serum and hepatic lipids in rats fed a high-fat diet. Biol Pharm Bull 17, 917-920.

21. Choi MS, Jung UJ, Kim HJ, et al. (2008) Du-zhong (Eucommia ulmoides Oliver) leaf extract mediates hypolipidemic action in hamsters fed a high-fat diet. Am J Chin Med 36, 81-93.

22. Lee MK, Kim MJ, Cho SY, et al. (2005) Hypoglycemic effect of Du-zhong (Eucommia ulmoides Oliv.) leaves in streptozotocin-induced diabetic rats. Diabetes Res Clin Pract 67, 22-28.

23. Park SA, Choi MS, Jung UJ, et al. (2006) Eucommia ulmoides Oliver leaf extract increases endogenous antioxidant activity in type 2 diabetic mice. J Med Food 9, 474-479.

24. Kavitha R \& Nalini N (2000) Hypolipidemic effect of green and red chilli extract in rats fed a high-fat diet. Med Sci Res 28, 17-21.

25. Palou A, Pico C, Bonet M, et al. (1998) The uncoupling protein, thermogenin. Int J Biochem Cell Biol 30, 7-11.

26. Samec S, Seydoux J \& Dulloo AG (1998) Role of UCP homologues in skeletal muscles and brown adipose tissue: mediator of thermogenesis or regulators of lipids as fuel substrate? FASEB $J$ 12, 715-724

27. Ricquier D, Thibault J, Bouillaud F, et al. (1983) Molecular approach to thermogenesis in brown adipose tissue. Cell-free translation of mRNA and characterization of the mitochondrial uncoupling protein. $J$ Biol Chem 258, 6675-6677.

28. Negre-Salvayre A, Hirtz C, Carrera G, et al. (1997) A role for uncoupling protein-2 as regulator of mitochondrial hydrogen peroxide generation. FASEB $J \mathbf{1 1}, 809-815$.

29. Vodal-Puig A, Solanes G, Grujic D, et al. (1997) UCP3: an uncoupling protein homologue expressed preferentially and abundantly in skeletal muscle and brown adipose tissue. Biochem Biophys Res Commun 235, 79-82.

30. Jiao K, Liu H, Chen J, et al. (2008) Roles of plasma interleukin6 and tumor necrosis factor-alpha and FFA and TG in the development of insulin resistance induced by high-fat diet. Cytokine 42, 161-169. 